Archives de sciences sociales des religions

142 | avril-juin 2008

Varia

\title{
La croyance comme ressource pratique
}

Olivier Tschannen

\section{OpenEdition}

Journals

Édition électronique

URL : http://journals.openedition.org/assr/14483

DOI : $10.4000 /$ assr. 14483

ISSN : $1777-5825$

Éditeur

Éditions de l'EHESS

Édition imprimée

Date de publication : 1 juin 2008

Pagination : 131-149

ISBN : 978-2-7132-2190-3

ISSN : 0335-5985

Référence électronique

Olivier Tschannen, "La croyance comme ressource pratique ", Archives de sciences sociales des religions [En ligne], 142 I avril-juin 2008, mis en ligne le 20 octobre 2011, consulté le 30 avril 2019. URL : http://journals.openedition.org/assr/14483 ; DOI : 10.4000/assr.14483 


\section{Olivier Tschannen}

\section{La croyance comme ressource pratique}

Il est devenu banal de dire que nous vivons dans une situation qu'on peut comparer à celle d'un "supermarché religieux ", sur lequel se rencontrent une " offre spirituelle» surabondante et une "demande de sens " de plus en plus éclatée $^{1}$. Il est vrai que l'idée d'appliquer à la religion ce vocabulaire, qui est celui de l'économie (Moore, 1994), heurte la conception que nous nous faisons généralement de la spiritualité. Pourtant, force est de constater que la métaphore du marché est très largement utilisée, aussi bien dans les publications universitaires que dans la presse. Et si cette métaphore connaît un tel succès, c'est certainement parce qu'elle est éloquente : pour se faire une idée de ce qu'est le supermarché religieux, il suffit d'entrer dans une librairie et de consulter les titres des ouvrages proposés au rayon spiritualité. Et lorsque, prolongeant l'analogie, on dit que face à cette "offre ", chacun de nous fait ses "choix " librement, et se compose ou se "bricole » une "religion à la carte ", cela se passe de commentaire : tout le monde a compris. Si donc le vocabulaire peut être contesté, il n'en reste pas moins qu'il reflète un sentiment largement répandu : c'est que les selfservices du religieux ont envahi nos existences un peu comme les hypermarchés ont envahi nos banlieues.

\section{L'individu comme usager}

En règle générale, dans la littérature sociologique qui traite de ces thèmes, le regard des chercheurs est nettement orienté vers l'offre, et tend à négliger la demande. On se pose avant tout la question de savoir comment la religion s'est réorganisée dans le monde moderne, plutôt que la question de savoir ce que les individus attendent de la religion, et surtout ce qu'ils en font. L'intérêt principal des chercheurs va à la compréhension des mécanismes sociaux du métissage, du

1. Cette réflexion est issue d'une HDR défendue à l'EHESS. Je remercie pour leur lecture exigeante et leurs commentaires généreux les membres du jury, en premier lieu Jean Widmer, dont l'apport intellectuel nous manque cruellement depuis qu'il a été prématurément enlevé à l'affection des siens, Danièle Hervieu-Léger (directrice), Karel Dobbelaere, Enzo Pace, Jean Séguy et Jean-Paul Willaime, ainsi que les trois lecteurs anonymes des ASSR. 
I32 - ARCHIVES DE SCIENCES SOCIALES DES RELIGIONS

syncrétisme, de la réinvention qui, dans le grand brassage planétaire, aboutissent à la mise en place d'une offre polyphonique, fragmentée, voire anarchique. C'est dans cette offre que s'inscrivent les choix de l'individu, et la manière dont il va «bricoler » sa religion personnelle. Mais comment le fait-il ? Pourquoi le fait-il ? Quel est le sens de ce bricolage pour lui ? Quelles en sont les conséquences sur sa vie? Ce sont des questions qui, le plus souvent, sont passées sous silence ou traitées comme accessoires. Comme si, à quelques exceptions près ${ }^{2}$, les sociologues n'avaient pas encore pris toute la mesure de la transformation - que pourtant ils ont eux-mêmes bien mise en évidence dans leurs études sur les mutations contemporaines de la religion - qui fait que désormais, pour comprendre les dynamiques du religieux, on ne peut plus partir du haut - c'est-à-dire de l'institution, de l'organisation, de la culture, de l'offre - mais qu'il faut au contraire partir du bas - c'est-à-dire de l'individu, de ses besoins, de sa vie concrète, bref de ses demandes en matière de croyances.

Je propose d'utiliser l'expression « individualisme croyant " pour caractériser, sous l'angle qui vient d'être précisé, le rapport que l'individu moderne entretient aux croyances. Au sens où je l'entends, l'individualisme croyant réfère à une situation où une personne utilise la croyance comme ressource pour résoudre un problème apparu dans sa trajectoire personnelle, l'idée de trajectoire renvoyant au processus par lequel la personne se construit comme sujet (Tschannen, 1998).

Le fait que j'utilise un vocabulaire à connotation pragmatique ("problème ", "ressource ") ne signifie pas que je nie la dimension symbolique de l'individualisme croyant. Je me situe dans le prolongement de la philosophie pragmatiste américaine, qui considère les idées comme des outils, ce qui permet de concevoir la notion de problème en un sens large : tout ce qui pose question et qui exige une solution, quel que soit le type de question et de solution, correspond à un "problème ». Ressources et trajectoires sont inséparables : un problème ne se pose comme tel que dans le cadre d'une trajectoire; ce qui "fait problème " pour telle personne ne fera pas forcément problème pour telle autre. Par ailleurs, les ressources ne sont pas forcément matérielles : il en est qui sont hautement symboliques. Par exemple, les théodicées, pièces maîtresses de toute théologie (Berger, 1967 : 53-80), constituent un excellent exemple de ressources religieuses : ce sont des constructions symboliques plongeant leurs racines dans une respectable tradition, qui permettent de répondre au " problème » du pourquoi de la souffrance.

2. Par exemple Hervieu-Léger (1999), qui propose une analyse des modalités de l'adhésion religieuse permettant de retracer la logique des trajectoires individuelles. Ou Kuhlman (1999), qui propose de considérer le New Age comme fournissant aux individus les moyens de s'adapter à une civilisation devenue "réflexive " en apprenant à mieux distinguer leurs fonctions psychiques. 


\section{Pluralisme culturel et quête de l'intériorité}

Mais revenons pour un instant à l'offre, c'est-à-dire aux croyances auxquelles l'individu se trouve exposé dans sa trajectoire. En référence à Thomas Luckmann, on peut dire que l'offre religieuse contemporaine est caractérisée par un niveau de transcendance relativement bas (1990). Comment expliquer cet effondrement de la transcendance - qui d'ailleurs semble constituer, à juste titre serait-on tenté de dire, la préoccupation principale du pape Benoît XVI ? Sociologiquement, cette tendance peut être interprétée comme une conséquence du pluralisme culturel. Dans la mesure où les croyances véhiculées par chaque groupe sont relativisées par leur confrontation aux vérités véhiculées par d'autres groupes, les grandes transcendances inscrites dans les cultures de ces groupes sont remises en question (Berger et Luckmann, 1966). À la limite, dans une situation de mondialisation, le seul garant possible de la vérité absolue dont est porteuse la religion est l'universel humain, auquel on pense avoir accès au fond de soi. Ce fait entraîne une conséquence remarquable : la réponse à la question du sens n'est plus cherchée dans la communauté, mais dans un universel humain dont on suppose la présence au fond de son être propre (New Age), éventuellement en interaction ponctuelle avec autrui, dans les "petits groupes ". C'est pourquoi à la fois les techniques psycho-corporelles, qui abolissent la transcendance entre le soi et l'inconscient, et les petits groupes, qui abolissent la transcendance entre soi et autrui, sont si importants dans la religiosité actuelle.

En un certain sens, l'individualisme croyant moderne est l'héritier de la religiosité des "virtuoses religieux " au sens de Weber, des saints et des mystiques, des générations d'ermites et de moines ayant fait vœu de silence; mais dans le même temps, et c'est certainement plus important, il est le produit d'une transformation de la religiosité ordinaire. L'adepte du Nouvel Âge qui cherche l'Universel au fond de soi n'obéit pas à une logique très différente de celle du fidèle ordinaire qui, dans l'Europe mi-chrétienne et mi-paienne du Moyen Âge, trouvait la Vérité dans la tradition dont était porteuse sa communauté. Dans les deux cas, comme le dirait Robin Horton, on cherche la Vérité là où la structure de la société à laquelle on appartient semble l'avoir placée $^{3}$. La réponse à laquelle aboutit la quête spirituelle individualiste moderne est d'abord le produit d'un âge où les cultures locales ont été relativisées au profit d'une Humanité abstraite, dont on pense trouver la marque au centre même de son être propre après un long cheminement personnel, par lequel on se construit comme sujet.

3. Ce raisonnement a probablement été inspiré à Horton (1993 [1960-1984]), par Elias (1993 [1983] : 95-99). 
I 34 - ARCHIVES DE SCIENCES SOCIALES DES RELIGIONS

\section{Individualisme et autonomie}

Cette idée d'un individu qui se construit comme sujet peut être considérée comme le mythe fondamental de l'individualisme moderne - un mythe devenu réalité sociale par le mécanisme de la prophétie auto-réalisatrice. Toutefois, cette nouvelle réalité sociale n'est pas un pur produit de la croyance : les transformations structurelles au fondement du mythe de l'individu possèdent bel et bien une réalité, qui ne doit rien aux croyances.

Pour clarifier ce point, je propose de revenir aux fondements classiques de la notion d'individualisme. Il s'agit d'éviter le piège qui consisterait à réduire l'individualisme au plus récent de ses avatars, par exemple celui dont a rendu compte Alain Ehrenberg dans Le culte de la performance (1991). Ehrenberg $\mathrm{y}$ met en scène un individu d'un type nouveau, produit des mutations socioéconomiques des années quatre-vingts, qui se définit dans une compétition acharnée dans tous les domaines de l'existence : sport, vie professionnelle, voire même consommation. Certes, en un sens, le choix d'une telle approche de l'individu pourrait apparaître comme plus que justifié : après tout, l'obligation d'être performant n'est-elle pas la manifestation du même esprit que celui qui pousse les bricoleurs religieux à se composer une panoplie aussi efficace que possible d'outils empruntés aux différentes traditions religieuses? On ne saurait le nier. Mais le problème, c'est qu'en prenant cette réalité pour point de départ, on se condamnerait à la retrouver telle quelle au point d'aboutissement de la recherche, en s'interdisant par là même de répondre à une question de fond: dans quelle mesure cet individualisme croyant est-il véritablement moderne (ou propre aux dernières décennies du $\mathrm{xx}^{\mathrm{e}}$ siècle) - ce qui conforterait la thèse du mythe -, et dans quelle mesure plonge-t-il au contraire ses racines beaucoup plus loin, confirmant ainsi l'idée d'un fondement social antérieur au mythe? Ou, pour formuler la question autrement, dans quelle mesure est-il proprement occidental, et dans quelle mesure est-il au contraire une figure universelle ?

C'est pourquoi, parmi les différentes approches de l'individualisme, j'ai choisi celle qui me paraît la plus large et générale. Cette conception trouve sa source chez Ferdinand Tönnies, et accessoirement chez Georg Simmel. Tönnies établit une distinction entre deux formes d'individualisme. La première est universelle, alors que la seconde est propre à ce qu'il appelle "société " (par opposition à la "communauté »). La première est "naturelle ", en ce sens que l'homme est " par nature " un individu, un être qui tendra à privilégier ses propres besoins et désirs sur ceux du monde environnant.

Il vaut la peine de s'arrêter un instant sur cette idée d'un individualisme " égoïste » (comme l'appelle Tönnies), dans la mesure où il s'agit d'une conception que la sociologie a combattu avec acharnement. Comme l'a montré Robert Nisbet (1984 [1966]), la naissance de la sociologie au XIX ${ }^{\mathrm{c}}$ siècle peut être interprétée comme une réaction aux idées individualistes du siècle précédent, telles 
qu'elles se sont exprimées dans la philosophie individualiste des Lumières et dans le courant utilitariste en économie politique. En France tout particulièrement, le courant "holiste " a longtemps dominé le champ sociologique de manière hégémonique, à telle enseigne que dans les années soixante encore, il n'était pas rare pour un jeune ethnologue "féru de sociologisme durkheimien, de structuralisme lévi-straussien, de culturalisme américain ou même d'anthropologie marxiste » de découvrir avec étonnement, «à participer à la vie concrète d'une modeste société villageoise (...) à quel point chaque individu y pouvait être marqué et reconnu très explicitement dans sa différence, non seulement par sa position sociale spécifique, mais aussi dans sa personnalité propre, ses traits de caractère et de comportement, ses talents, ses qualités et ses défauts ${ }^{4}$. Aujourd'hui encore, on trouve dans certains manuels de sociologie l'idée selon laquelle dans les sociétés tribales, le soi individuel n'existerait pas. C'est le cas par exemple dans tel traité post-moderniste ${ }^{5}$, où l'on peut lire que dans certaines tribus, les individus auraient si peu conscience de leur identité propre que lorsque l'un d'entre eux meurt, les autres ne semblent pas se rendre compte de son absence. L'exemple peut sembler extrême : il n'empêche qu'il a été publié, en 2000, par Oxford University Press dans un manuel de sociologie dont on peut être certain qu'il a été et sera lu par des milliers d'étudiants.

Tönnies, comme les sociologues allemands de manière générale d'ailleurs, n'avait pas ces œillères. Selon lui, il existe bel et bien un individualisme " égoïste " universel, pré-social. Toutefois, celui-ci varie selon la position sociale : "Dans chaque culture il existe des personnes plus "individuelles" et moins individuelles. Ainsi par exemple les maîtres sont plus individuels que les serviteurs, les hommes plus individuels que les femmes, les citadins plus individuels que les campagnards » (Kippele, $1998: 55)^{6}$. Pour qualifier ce type d'individualisme, Tönnies utilise l'expression « individualisme dans les liens sociaux ", qui réfère au fait que l'individu cherche à réaliser son intérêt sans pour autant s'extraire des relations communautaires dans lesquelles il est pris, puisque c'est au contraire à travers celles-ci qu'il se réalise, comme le montre bien le fait que ce sont surtout les dominants qui ont la possibilité de s'affirmer. La seconde forme d'individualisme, condensée dans l'expression « individualisme hors des liens sociaux ", est celle qui est vraiment nouvelle, et propre à la "société »: "Ce n'est pas la tendance à la différenciation individuelle et à la centration sur soi ("égoïsme") qui est nouvelle, mais l'émancipation par rapport aux liens traditionnels "

4. J'emprunte cette citation à l'africaniste Marie. Elle se trouve à la page 279 du rapport polycopié qui a servi de base à la fabrication du livre publié sous sa direction (1997).

5. Il s'agit du traité de Holstein et Gubrium (2000). Le passage cité est basé sur l'ahurissant récit « ethnographique » de Schneebaum (1969), qui était un véritable best-seller à l'époque de la contre-culture.

6. Pour Tönnies et Simmel, je me suis beaucoup appuyé sur Kippele, qui s'est livrée à une lecture très minutieuse du thème de l'individualisme chez les principaux auteurs classiques de la sociologie. 
I36 - ARCHIVES DE SCIENCES SOCIALES DES RELIGIONS

(Kippele, 1998 : 60). L'individu devient alors un atome isolé, apte à entrer dans de nouvelles relations, de type sociétaire.

L'évolution décrite par Tönnies pourrait être illustrée par une multitude d'exemples historiques. Elle s'exprime par exemple dans ce genre littéraire qu'est le roman de formation, qui montre qu'il est désormais possible, " en cette période de grande mobilité sociale que représente le XVIII ${ }^{\mathrm{e}}$ siècle, d'échapper à sa classe d'origine en intégrant les sphères de l'argent ou du pouvoir ». Dans la vie du héros, ceci se traduit souvent par le « refus du fils d'accepter un statut imposé par sa naissance, et (...) la volonté d'y échapper en rompant avec son pays d'origine » (Bancaud-Maënen, 1998 : 50, 56). Aux États-Unis, à la même époque, comme l'ont montré Robert Bellah et son équipe, ce refus est même devenu un impératif social : il existe une tradition américaine du "leaving home " (Bellah et al., 1985 : 56-62). La personne n'est considérée comme véritablement adulte qu'une fois qu'elle a rompu (fût-ce de manière théâtrale et provisoire) avec le milieu dans lequel elle est née.

Cette conception de l'individualisme est proche de celle développée par Simmel dans sa sociologie formelle. Avec la croissance en taille des groupes et leur différenciation interne, la relation entre l'individu et son groupe d'appartenance se distend. L'individu s'engage moins fortement en faveur de son groupe ; inversement, il est de plus en plus contraint de se prendre en charge lui-même. Mais dans le même temps, il multipliera les attaches sociales, dont chacune est plus partielle parce que plus spécialisée, mais dont la somme le lie encore plus étroitement à la société. Si donc l'individu s'autonomise, cela ne signifie pas qu'il se coupe de toute dépendance sociale, au contraire. C'est justement cette multiplication des attaches sociales qui rend possible l'individualisation. Si l'individu s'autonomise, c'est parce qu'il n'est plus dépendant d'aucune personne ni groupe spécifique : "même si globalement - quand on prend en considération la somme de tous les liens - chaque personne est beaucoup plus dépendante, elle est néanmoins moins dépendante des personnes particulières. La société devient plus dense, et les hommes plus individuels » (Kippele, 1998 : 73). L'individualisme produit par ces transformations prend deux formes bien distinctes. La première est celle de la "liberté par rapport aux attaches " (Ungebundenheit bedeutet Freiheit von etwas "), la seconde celle de la «liberté pour quelque chose» («Einzigartigkeit bedeutet Freiheit für etwas»).

Il semble que l'évolution historique oblige à combiner ces deux logiques. Ainsi, à la Renaissance, avec ce que Simmel appelle "l'individualisme de la distinction ", l'individu se réalise, comme le dirait Tönnies, «dans les liens sociaux ", c'est-à-dire à travers "sa volonté de puissance, son désir de se distinguer et d'être honoré » (Simmel, cité in Michon, 1999: 52). Au XVIII ${ }^{\mathrm{e}}$ siècle au contraire, l'individualisme est conçu comme une forme de liberté par laquelle chacun découvre en lui des attributs humains universels : "L'individu concret 
est négligé au profit de l'essence humaine qu'il incarne. Contrairement à l'individu de la Renaissance, sa spécificité, sa différence n'ont pas d'importance " (Michon, 1999 : 52). Finalement, à l'époque du romantisme, l'individualisme est conçu comme une forme de liberté permettant à chacun de découvrir en lui une altérité radicale: "L'individu était valorisé sur la base du fait qu'il était impossible à confondre avec un autre [Aufgrund seiner Unverwechselbarkeit] et sur la base de sa différence intérieure [seiner inneren Ungleichheit] » (Kippele, 1998 : 74).

Le balancement historique des formes de l'individualisme oblige donc à combiner deux critères : celui de l'autonomisation (Ungebundenheit) d'une part, et celui de la recherche de l'originalité (Einzigartigkeit) d'autre part. On peut chercher à être original (c'est-à-dire " différent ») dans les liens sociaux, comme à la Renaissance, ou hors des liens sociaux, comme à l'époque romantique. En fait, entre l'individualisme de la Renaissance et celui des Romantiques, on trouve une ressemblance: dans les deux cas, il s'agit de se distinguer de la "masse " par une sorte de prise de distance " aristocratique ", bien différente de l'attitude $\mathrm{du}$ philosophe des Lumières, qui se veut " démocratique » dans son appel à une raison universellement partagée.

Pour la suite, en combinant ces deux critères, je retiendrai de Tönnies et de Simmel l'idée que l'individu au sens moderne est le produit à la fois d'une rupture avec la communauté, c'est-à-dire d'une autonomisation par rapport au milieu socialisateur, et d'une projection vers un avenir conçu comme personnel, avenir qui passe par l'établissement de nouveaux liens sociaux, choisis en fonction de ce projet personnel. Par la suite, on verra que dans l'individualisme croyant moderne, ces liens sociaux nouveaux prennent souvent la forme de la participation à un " petit groupe ».

Pour faire apparaître la spécificité de cette conception, on peut la contraster avec d'autres définitions de l'individualisme, plus usuelles en sociologie. En effet, l'individualisme est généralement considéré comme le produit d'une construction sociale. En ce sens, l'individualisme est une forme de conformité, puisque - en particulier dans la société moderne - la personne qui s'individualise répond à la norme sociale de valorisation de l'individu. L'individualisme est socialement prescrit : c'est le point de vue adopté par Ehrenberg (1991 : 21), qui se situe par là même dans une perspective on ne peut plus classique. Durkheim, surtout, avait insisté sur ce point : "Nul ne conteste aujourd'hui le caractère obligatoire de la règle qui nous ordonne d'être, et d'être de plus en plus, une personne » (1930 [1893] : 401). Et Dumont, qui se situe dans la même filiation, renchérit : "Notre société nous fait une obligation d'être libres" (1966:21). Cette inversion de la logique du sens commun correspond bien à une certaine vocation que s'est donnée la sociologie : montrer que ce qui apparaît, au regard non informé, comme l'expression de réalités psychologiques ou de la liberté humaine, doit en réalité être compris comme le produit de conditionnements sociaux. 
I 38 - ARChives DE SCIENCES SOCIALES DES RELIGIONS

Mais il ne suffit pas, en s'inspirant de Tönnies, de définir l'individualisme comme une forme de rupture avec le milieu socialisateur pour échapper à cette logique d'inversion. En effet, cette rupture peut obéir à au moins trois logiques différentes, dont les deux premières ne sont rien d'autre qu'une retraduction du postulat durkheimien du primat du social. La première est justement celle de l'inversion : ainsi, dans certains cas, la déviance peut être considérée comme une simple conformité "à rebours " - c'est en tout cas l'argument qu'on oppose souvent aux révoltes adolescentes. La deuxième logique, très présente dans la littérature sociologique, est celle de la rupture avec le milieu socialisateur par conformité avec un nouveau milieu d'appartenance. Ce cas de figure correspond aux cas, bien connus, de la conformité à un milieu déviant, de la socialisation anticipée, de la migration et de la conversion. Dans ces deux premières logiques, on ne fait à nouveau que vérifier une même "loi " sociologique, celle de la conformité, à laquelle on ne peut échapper que par une inversion de cette conformité, ou par une autre conformité.

La troisième logique, qui est celle qui m'intéresse, serait celle qui permettrait une rupture accompagnée d'une construction, par l'individu en rupture, d'un type d'identité et de rapport au monde qui ne correspondent pas directement à un modèle social préexistant - même s'il est évidemment construit à partir d'ingrédients trouvés dans des modèles socialement donnés. Il s'agirait donc d'une situation dans laquelle la personnalité se construirait contre la conformité sociale et malgré le poids de celle-ci, où se formerait en elle un habitus qui serait le produit de la résistance à la structure sociale d'origine, plutôt que d'en être la reproduction, et sans en être la simple inversion.

\section{Religion et individualisme}

Mais revenons à la forme "croyante » de l'individualisme. Selon Danièle Hervieu-Léger, " on peut parler d'individualisation du religieux dès lors qu'intervient la différenciation entre une religion rituelle, laquelle requiert uniquement des fidèles l'observation minutieuse des pratiques prescrites, et une religion de l'intériorité qui implique, sur le mode mystique ou éthique, l'appropriation personnelle des vérités religieuses par chaque croyant. Dans toutes les grandes religions, cette différenciation s'est manifestée sous des formes diverses, bien avant l'émergence de la modernité » (1999: 157-158).

Pour comprendre la spécificité de ce point de vue, il faut à nouveau procéder par comparaison, en montrant quelle place il occupe dans l'univers des définitions possibles de l'individualisme croyant. Analytiquement, on peut distinguer plusieurs critères liés à un usage possible de ce concept. Le premier est la notion de choix. Toute croyance ou pratique qui résulte d'un libre choix individuel peut être considérée comme la manifestation d'une forme d'individualisme croyant. 
Cette acception du terme désigne donc une manière d'accéder à la religion : par choix personnel plutôt que par imposition sociale. Dans cette acception, l'individualisme croyant est le rapport au religieux le plus typique de la modernité, si l'on admet que « dans une société "sortie de la religion", il n'est (au moins tendanciellement) de croyant religieux que volontaire et de communalisation religieuse que choisie » (Hervieu-Léger, 2001 : 171). Une deuxième signification possible renvoie, non pas à la manière d'accéder à la religion, mais à son contenu même. Par exemple, une religion qui met l'accent sur l'accomplissement de soimême plutôt que sur la conformité sociale peut être considérée comme individualiste. En ce sens, la dimension individualiste peut aussi concerner les pratiques, et désigner une religion qui se pratique en solitaire plutôt que collectivement. Enfin, la notion d'individualisme peut référer au fondement des croyances. Ainsi, une religion qui affirme que la vérité n'est pas entièrement inscrite dans un texte ou une tradition, mais doit être découverte par un cheminement personnel, peut être dite individualiste.

Le fait de choisir une religion pour résoudre un problème ne signale donc pas forcément une position d'individualisme croyant. On connaît des sectes qui, pour avoir été choisies par leurs adeptes, n'en exigent pas moins une forme de soumission qui n'est guère compatible avec l'individualisme. Pour qu'on puisse parler d'individualisme croyant, il faut que la personne se soit appropriée la religion de manière suffisamment personnelle pour déboucher sur une trajectoire permettant l'émergence d'un sujet. Tel est bien le cas, par exemple, chez le renonçant hindou: "L'homme qui cherche la vérité ultime abandonne la vie sociale et ses contraintes pour se consacrer à son progrès et à sa destinée propres (...) la découverte de soi se confond pour lui (...) avec la libération des entraves de la vie telle qu'elle est vécue dans ce monde " (Dumont, 1983:35). Tel est aussi le cas dans l'histoire de la mystique chrétienne, qui peut même être entièrement lue comme "une histoire de la construction du sujet religieux » (Hervieu-Léger, 1999 : 158). Dans l'Europe médiévale déjà, une inscription gravée vers 1070 sur le portique d'une église proclamait : "You'll climb to heaven if you know yourself " ${ }^{7}$. Dans les religiosités mystiques, obtenir le salut, c'est se connaître soi-même, c'est-à-dire émerger, à ses propres yeux, comme un sujet.

\section{Illustration}

Pour illustrer cette conception de l'individualisme croyant, je m'appuierai sur quelques cas tirés de l'étude de Frédéric Lenoir sur le bouddhisme en France (1999). Cette manière de procéder est destinée à la fois à exemplifier la notion

7. Et Morris, qui rapporte cette inscription, renchérit : «It would indeed be scarcely too much to describe the eremetical movement as a revolt of individuals against a constricting society » (1987 [1972] : 32). 
I4O - ARCHIVES DE SCIENCES SOCIALES DES RELIGIONS

et à en approfondir l'analyse ${ }^{8}$. Pour clarifier le statut méthodologique de ma démarche, je dirais que je traiterai les personnes dont les biographies sont présentées par Lenoir comme des personnages littéraires, c'est-à-dire comme des êtres de papier entièrement contenus dans les pages qui les décrivent, comme le sont les personnages de romans.

Les quatre cas que je présente (parmi les dix de Lenoir) sont destinés à illustrer, de manière aussi condensée que possible, les idées suivantes. Premièrement, chacune des personnes interviewées par Lenoir utilise le bouddhisme pour résoudre un problème ou, plus largement, trouver des solutions à une situation complexe faite d'un ensemble de problèmes. Deuxièmement, chacune de ces personnes découvre le bouddhisme à travers une trajectoire propre. Et troisièmement, pour résoudre son problème, elle s'approprie le bouddhisme d'une manière qui est assez personnelle. C'est pourquoi chaque cas sera présenté comme une suite de trois épisodes: problème/trajectoire/solution, qui sera suivie par une courte analyse de la pertinence de l'exemple choisi au regard des critères donnés plus haut. À noter que la question de la rupture, à laquelle j'ai donné une certaine importance dans ma définition de l'individualisme, n'apparaît pas comme un de ces épisodes. Pourtant, on verra qu'elle est bel et bien présente, sauf dans le dernier cas, dont je me saisirai d'ailleurs comme d'une occasion pour approfondir cette question.

Premier cas : chez Jacqueline, 65 ans, artiste peintre et professeur de lettres à la retraite, le bouddhisme apparaît comme un moyen de trouver le bonheur en surmontant les séquelles d'une éducation catholique mutilante. Pour elle, «le but du bouddhisme, c'est le bonheur " ${ }^{9}$, en contraste total avec ce que lui avait fait vivre le catholicisme : "C’est la culpabilité judéo-chrétienne qui m'a empêchée de vivre pendant des décennies ».

Si Jacqueline, comme de nombreux occidentaux, médit du catholicisme de son enfance, il ne faut bien sûr pas en chercher les raisons dans le catholicisme en tant que tel ${ }^{10}$, mais dans les circonstances historiques qui ont simultanément produit un certain type de catholicisme et une montée massive d'aspirations en contradiction avec celui-ci (Pelletier, 2002). Ce qui est le cas chez Jacqueline : elle a suivi sa scolarité dans un internat catholique, dans un climat qu'elle décrit

8. À noter que je restitue les entretiens recueillis par Lenoir en suivant une logique différente de la sienne.

9. Toutes les citations dans les quatre cas qui suivent sont tirées de l'étude de Lenoir (1999: 143-210).

10. Il serait abusif d'établir une correspondance nécessaire entre catholicisme et malheur, ou d'ailleurs entre bouddhisme et bonheur. À preuve, les mots de cette réfugiée cambodgienne convertie au catholicisme : "L'action humanitaire, l'éducation, la façon de considérer les gens et de respecter leur dignité, dans tout cela je sentais les racines chrétiennes et je me disais que le christianisme donnait de bons fruits. Alors que, pour moi, malgré sa sagesse, le bouddhisme insiste trop sur la souffrance "qui suit l'homme comme son ombre" : on la subit, on ne peut ni s'en débarrasser ni lui donner un sens spirituel »(de Lagarde, 1996 : 73-74). 
comme dominé de manière permanente par l'obsession du péché. Toute sa trajectoire peut se comprendre comme un effort pour échapper aux conséquences mutilantes de cette prime éducation d'une part, et à l'enfermement dans son milieu ouvrier d'origine d'autre part : "J'avais un appétit féroce de dépasser le milieu ouvrier dans lequel je vivais, qui était extrêmement fermé sur lui-même ". C'est pourquoi, à coups de privations, elle choisit la voie des études pour devenir professeur de lettres. Ces deux aspirations - les études et la spiritualité - sont d'ailleurs clairement liées dans son récit : "Comme nourriture spirituelle, à la bibliothèque de Cherbourg, j'aimais beaucoup lire ». C'est à la bibliothèque qu'elle découvre le poète indien Tagore, qui lui révèle que Dieu peut se manifester d'une manière radicalement opposée à celle qu'elle a vécue dans son enfance : "Je me souviens encore de l'un de ses vers: Que mon être soit une flûte de rosean que tu puisses emplir de ta musique. (...) j'ai eu un choc spirituel le jour où j'ai su que Dieu pouvait passer à travers nous et chanter ». Pourtant, jusqu'à l'âge de quarante-huit ans, elle laissera ses aspirations spirituelles en sommeil : grande lectrice de Nietzsche, athée convaincue, militante de gauche et de la cause féministe, elle vit tous les événements de sa vie (deux mariages, un enfant, des dépressions, sa fonction de directrice d'école, sa découverte de la peinture) comme un combat. Ceci jusqu'au jour où, alors qu'elle visite le centre bouddhiste de Karma Ling, se fait enfin la révélation, par l'intermédiaire de lama Denys Teundroup : "Cette conférence m'a vraiment ébranlée; à chaque phrase que prononçait lama Denys, je me disais: "c'est juste”. Chaque enseignement, chaque fragment d'enseignement tombait juste pour l'intelligence et le cœur. Cela, je ne l'avais jamais vécu ». C'est à ce moment qu'entourée par un groupe de gens "en quête " comme elle, qui lui permettent pour la première fois de communiquer de manière satisfaisante, elle " entre dans la vie de l'esprit ».

Le cas de Jacqueline est l'occasion d'illustrer l'importance, annoncée plus haut, des "petits groupes » dans l'individualisme croyant moderne. Comme l'a montré Hervieu-Léger, ceux-ci fournissent une forme de "validation " de la croyance religieuse qui se situe en quelque sorte à mi-chemin entre la validation « institutionnelle », rejetée parce que trop contraignante (comme l'est la validation " communautaire »), et l' " autovalidation », délaissée parce qu'insuffisante. En joignant un petit groupe, l'individualiste croyant moderne entre dans le régime de la "validation mutuelle du croire ", dont on aurait d'ailleurs tort de penser qu'il fonctionne exclusivement dans les religions de type oriental : "Un bon exemple en est donné par les groupes spirituels, catholique ou protestant, qui réunissent, de façon le plus souvent informelle, des professionnels œuvrant dans le même secteur d'activité (...). Leur préoccupation principale (...) est (...) de donner à chacun les conditions optimales d'une expression de ses expériences et de ses attentes »(Hervieu-Léger, 1999: 182).

Par ailleurs, dans la mesure où Jacqueline est l'une des trois personnes classées par Lenoir dans la catégorie de ceux qui ont renié leur religion d'origine et se 
définissent désormais comme bouddhistes, il faut affronter directement la question de savoir dans quelle mesure sa conversion est autre chose qu'un nouveau conformisme. Le cas de Jacqueline peut-il être considéré comme une illustration de ce que je cherche : des personnes qui se construisent comme sujet ? La question est délicate et, en un sens, elle ne peut pas être tranchée de manière certaine. Mais ce qui m'intéresse, ce n'est pas tellement de décider si Jacqueline s'est construite comme sujet ou non - d'autant qu'elle n'est pour moi qu'un être de papier. Ce qui m'intéresse, c'est d'utiliser ce cas concret pour affiner les critères définissant l'individualisme croyant au niveau conceptuel.

Avant toute chose, il faut prendre en considération la trajectoire de Jacqueline dans son ensemble, et ne pas oublier son âge. Pendant vingt-cinq ans, elle a mené ce qu'elle décrit comme " deux vies parallèles " : farouchement athée et militante, et en même temps engagée dans une quête spirituelle secrète, qu'elle sublimera en partie en se lançant dans la peinture. Avec un tel parcours, on ne l'imagine guère basculer d'un seul coup dans la soumission à une orthodoxie. C'est ce que confirme son attitude après sa conversion. Après sa prise de refuge, à quarantehuit ans, elle ne change rien à sa vie ordinaire : elle continue à assumer les huit cents élèves de sa "cité difficile ", elle continue à peindre, et partagera la vie de son mari, artiste-peintre, jusqu'à leur divorce qui se produit lorsqu'elle a soixante ans. Ses enfants et son petit-fils semblent très présents dans sa vie. Par ailleurs, elle ne prend pas comme lama-racine celui qui l'a convertie, mais prend le temps de visiter d'autres centres, et finit par choisir (on ne peut s'empêcher de penser à une sorte de réflexe d'indépendance) un lama qui n'est plus de ce monde.

Pourtant, on peut aussi porter sur la conversion de Jacqueline un regard différent. La structure classique du récit de conversion est bel et bien présente : pendant vingt-cinq ans, elle a cherché en vain ce qui lui permettrait de "transmuer sa vie ». À partir du moment où elle l'a trouvé, tout a changé : « Le travail du bouddhisme m'a permis cette mutation du non-sens au sens. Aujourd'hui je mets du sens partout, y compris dans les choses les plus modestes du quotidien. Je ne crois plus du tout au hasard ». Elle insiste beaucoup sur la nécessité d'échapper au "papillonnage ", d'approfondir sa voie : "Je ne suis pas du tout favorable au syncrétisme. Autant il est indispensable pour tout être humain d'expérimenter, (...) autant, à partir du moment où on a trouvé une voie juste par rapport à ce qu'on est, il est indispensable (...) d'entrer dans la voie profonde qu'on s'est choisie et de la pratiquer au maximum ».

Comment interpréter ce cas? On peut dire que la religion a acquis, dans l'architecture de la vie de Jacqueline, une place qui est centrale, mais qui semblait jusqu'alors inoccupée. À quarante-huit ans, sa vie était, à peu de chose près, structurée définitivement : une expérience riche, des lectures et des engagements, sa profession, sa passion pour la peinture, et une vie de famille derrière elle. Pourtant, une place structurellement centrale, celle du sens, restait vide. La conversion au bouddhisme n'a pas changé l'organisation de sa vie, mais est venue 
lui donner le sens qui lui manquait: désormais, à partir de ce centre, tout ce qu'elle vit prend sens. Ce n'est pas le bouddhisme qui a structuré la trajectoire de Jacqueline ; c'est au contraire sa trajectoire qui a fait qu'il existait, au centre de son existence, une place vide que le bouddhisme n'a eu qu'à occuper. Donc en un sens, au moment de la conversion de Jacqueline, tout a changé ; et pourtant en même temps, rien n'a changé. Jacqueline peut donc être considérée comme une illustration accomplie de ce que j'appelle l'individualisme croyant.

Deuxième cas : chez Christophe, 32 ans, RMIste, le bouddhisme apparaît comme un moyen de gérer sa vie de déclassé. Il reconnaît que lorsqu'il avait vingt ans, il avait en lui beaucoup d'agressivité et de violence, et que le bouddhisme lui a permis de relativiser ses sources d'amertume, même si occasionnellement celleci refait surface : "Pour ce qui est de l'attachement aux choses, il m'arrive d'avoir une pensée de jalousie dans la rue, de voir quelqu'un avec une belle voiture et de me dire : “Ah ce qu'il me fait chier, celui-là, avec sa BMW !" J'éprouve un peu de jalousie, mais après, j'ai de la compassion pour lui, parce que je me dis qu'il est complètement pris dans le monde, dans le samsâra ». Et c'est ce refus du matérialisme (qui, étant donné l'impossibilité pratique d'être matérialiste dans sa situation de RMIste, ressemble fort à une rationalisation) qui aura motivé son adhésion au bouddhisme : "Je n'avais plus vraiment d'illusions sur la société matérialiste et j'avais bien compris que c'était une grosse bouffonnerie. (...) la société marchande me sortait par les yeux ».

Christophe a suivi l'exemple de sa mère qui, après une longue recherche spirituelle ayant notamment passé par le soufisme, s'est finalement convertie au bouddhisme alors que lui-même avait vingt ans. Pendant ses études d'ingénieur, il constate que certains astrophysiciens sont eux-mêmes bouddhistes, et en conclut qu'il n'y a pas d'incompatibilité entre une approche scientifique et le bouddhisme. Quelques années plus tard, c'est avec sa mère qu'il rencontre pour la première fois un lama, auprès duquel il prend immédiatement refuge. De lama en lama (il en rencontrera plusieurs, qui tous l'ont influencé) et de voyage en voyage (au Tibet, au Népal, en Inde, en pèlerinage sur les lieux saints) il se convainc de plus en plus de la pertinence et de la justesse du bouddhisme.

Le bouddhisme auquel adhère Christophe est un curieux mélange de philosophie rationnelle et de croyance en la magie : pour lui, le bouddhisme est une démarche " extrêmement rationnelle, logique, totalement scientifique "; mais en même temps, il est convaincu que les grands lamas sont capables d'accomplir des miracles (il affirme en avoir été témoin) et cette aura magique contribue beaucoup à son attirance. Ces croyances lui permettent de vivre un quotidien (par la lecture, la méditation, la fréquentation des centres bouddhistes) qui nie et annihile en quelque sorte le monde matérialiste dans lequel il ne veut pas - ou ne peut pas - pénétrer.

Dans le cas de Christophe se repose la même question qu'avec Jacqueline, puisque lui aussi a été classé par Lenoir dans la catégorie de ceux qui disent 
adhérer complètement au bouddhisme. Mais dans son cas, les doutes quant à sa capacité à s'être construit comme sujet sont plus marqués. En effet, on ne peut pas dire que sa vie ait été structurée fermement avant son entrée dans le bouddhisme, au contraire : c'est sa mère qui l'y a initié, il n'a jamais travaillé, et depuis l'âge de vingt ans, le bouddhisme occupe une place structurante dans sa vie. D'ailleurs, son quotidien est entièrement organisé par le bouddhisme : il médite quotidiennement, fréquente un centre une fois par semaine, mais surtout, passe l'essentiel de son temps «à lire des livres de science et de spiritualité ». Le bouddhisme est non seulement au centre de son quotidien, c'est aussi son seul horizon pour l'avenir : il ne projette pas de travailler, se contentant du RMI, et ne mentionne pas de projets familiaux ; par contre, il projette de faire, dès qu'il se sentira prêt, plusieurs "grandes retraites " de trois ans, trois mois et trois jours : " Je pense que je vais faire plusieurs années de retraite en cette vie, c'est la seule chose vraiment importante».

Le cas de Christophe doit donc être considéré comme un contre-exemple. Malgré le fait qu'il vive une vie qui semble assez solitaire, malgré le fait que son bouddhisme soit une construction en partie assez personnelle, avec sa manière d'y intégrer sa formation d'ingénieur et ses lectures de vulgarisation scientifique, malgré le fait que sa conversion soit le produit d'un choix personnel, et malgré le fait qu'il ait utilisé le bouddhisme comme ressource pour résoudre sa situation personnelle, on ne peut pas parler d'individualisme croyant: son rapport au bouddhisme apparait comme une forme de conformisme inversé, un antimatérialisme réactionnel qui imprègne la totalité de son existence. Au fond, Christophe apparaît comme déterminé socialement par sa situation de RMIste : jusque dans sa valorisation religieuse de sa formation d'ingénieur impossible à valoriser sur la marché du travail, son système de croyances est le reflet inversé de sa situation sociale.

Troisième cas : chez Marie, 53 ans, psychologue, le bouddhisme apparaît, paradoxalement, comme une voie lui permettant d'affermir sa foi chrétienne. Ce qu'elle cherche, ce sont des moyens lui permettant de pallier certaines insuffisances de l'institution catholique, notamment du côté de « la participation active du corps».

Née dans un milieu catholique très pratiquant, animée depuis toujours d'une foi forte qui n'a jamais vacillé, Marie sent pourtant progressivement s'installer en elle un rejet de l'Église, qui se transforme en cassure le jour où elle découvre l'encyclique de Paul VI sur la contraception. Ce qui ne l'empêchera pas de rester catholique pratiquante, au nom de l'Évangile et du rapport à Dieu, qui est d'une importance fondamentale pour elle ("Je me sens très profondément fille de Dieu »), sans rien chercher ailleurs : «En fait, je n'avais pas de quête. J'avais des inconforts ». Pourtant, à la suite d'une crise personnelle, une " grande dépression ", elle rencontre un prêtre catholique pratiquant le bouddhisme zen, qui 
l'initie à la méditation. Plus tard, elle se rapproche du bouddhisme tibétain, qui lui procure un approfondissement spirituel.

Le bouddhisme a donc permis un renouvellement de la foi pour Marie. Ce constat soulève une question importante : à partir de quel moment peut-on parler de « rupture avec le milieu socialisateur » ? Culturellement, Marie est restée chrétienne, cela ne fait aucun doute; rien n'indique une réelle conversion au bouddhisme. Cependant, il ne faudrait pas se laisser aveugler par ces déclarations de fidélité chrétienne. La fidélité au Christ n'est pas la fidélité à l'Église. Or, sociologiquement, le milieu socialisateur est bel et bien l'Église. Et par rapport à celle-ci, il y a incontestablement rupture : d'ailleurs, elle préfère aujourd'hui se dire chrétienne que catholique, en ajoutant même : « de sensibilité protestante plutôt que catholique». En conclusion, on peut dire que Marie incarne une forme particulière d'individualisme croyant, dans lequel une pratique religieuse nouvelle permet de résoudre certains problèmes liés à des insatisfactions face à l'institution religieuse d'origine, sans pour autant induire de rupture avec le contenu même de la religion d'origine.

Quatrième cas : pour Pauline, 37 ans, psychologue, le bouddhisme constitue l'aboutissement d'une longue quête de "quelque chose " à l'ombre de la mort. Dans son enfance déjà, Pauline " cherchait quelque chose ", ce qui l'avait amenée à lire des vies du Bouddha et du Christ. Mais cette quête a pris une tournure nettement plus aiguë suite à un événement dramatique, dont elle parle comme "la grande chose de sa vie ": le décès de son ami, dont elle avait partagé la vie depuis l'âge de dix-sept ans, mort d'un cancer après sept ans de vie commune. En cela, elle est d'ailleurs représentative d'une tendance générale : on sait que les drames personnels sont une des causes les plus communes de l'émergence d'une demande religieuse (Hervieu-Léger, 1999: 131). Ce drame l'a précipitée dans un tourbillon d'expériences religieuses (retraite dans un cloître, stage Nouvel Âge, tarot ésotérique, méditation transcendantale). Dans un stage bouddhiste, des moines lui font remarquer que cette manière de papillonner ne menait à rien, et qu'il lui faudrait choisir. C'est au moment où elle suit un stage sur le Livre des morts tibétains (thème évidemment central pour elle : elle fait un mémoire de psychologie sur ce thème, et accompagne des mourants) que se fait la révélation.

Toutefois, sa vie ne semble pas centrée sur le bouddhisme, mais bel et bien sur le thème de la mort. Et comme elle le dit elle-même, pour gérer ce problème de la mort, elle met en œuvre une combinaison de trois pratiques : "Ces trois choses-là vont ensemble dans ma vie et se combinent: la psychothérapie, le bouddhisme et ce travail avec les mourants ». C'est la psychothérapie qu'elle suit régulièrement, et non le bouddhisme, qui lui a livré ce qu'elle considère comme la clé de lecture la plus générale de sa vie : elle dit savoir depuis là que la quête spirituelle qu'elle poursuit depuis son plus jeune âge est liée à " une blessure narcissique, quelque chose qui a manqué dans les premiers mois de la 
I 46 - ARCHIVES DE SCIENCES SOCIALES DES RELIGIONS

vie ou pendant la naissance ». Et c'est son travail avec les mourants, et non le bouddhisme, qui lui a récemment permis de dépasser les séquelles de cette blessure : "A un moment donné, mon intellect a tout lâché. Le cœur s'est ouvert. Cela s'est passé avec des femmes que j'ai accompagnées dans la mort. (...) Des gens me disaient que j'étais comme un rayon de soleil, qu'ils attendaient ma visite, que j'étais si douce, alors qu'on m'avait toujours dit l'inverse : que j'étais cassante, que je recelais une certaine violence ».

Dans le cas de Pauline, le bouddhisme apparaît comme un outil parmi d'autres dans une panoplie d'outils utilisés pour résoudre un problème personnel fondamental, qui se cristallise autour du thème de la mort. Pauline apparaît donc comme un bon exemple d'individualisme croyant.

Le cas de Pauline permet de revenir sur la question de la rupture avec le milieu socialisateur. Dans son cas, on ne voit guère de volonté de rupture; par contraste, dans les trois premiers cas, celle-ci était à chaque fois présente : rupture avec l'éducation catholique et ouvrière pour Jacqueline, avec la société de consommation pour Christophe, et avec l'Église catholique (l'encyclique sur la contraception) pour Marie.

Cette énumération suscite une interrogation : par rapport à quoi se fait la rupture ? Que signifie au juste "rupture avec le milieu socialisateur " ? S'agit-il de rompre avec les personnes ? Ou avec les valeurs dont ce milieu est porteur ? Ou encore avec l'habitus qui y a été inculqué ? Le cas de Marie a montré qu'il peut y avoir rupture avec les formes explicites de la socialisation, sans pour autant qu'il y ait rupture - bien au contraire - avec son contenu implicite (l'habitus), ni avec les personnes de ce milieu.

Qu'en est-il maintenant du cas de Pauline? Bien loin de revendiquer une quelconque rupture, elle se situe dans le prolongement d'une quête qu'elle fait remonter à sa naissance. Sa biographie ne se présente pas comme celle d'une conversion, au contraire : c'est la biographie de quelqu'un qui assemble pièce à pièce les morceaux du puzzle de sa vie (le Livre des morts tibétain, le mémoire de psychologie sur la mort, l'accompagnement des mourants, la psychothérapie), comme pour former une image qui aurait été prédéterminée dès sa naissance.

Si pourtant elle n'a pas vécu de rupture dans sa vie, il y a bien eu une cassure - c'est-à-dire une rupture subie plutôt que provoquée. Et c'est effectivement la mort de son ami qui, dit-elle, l'a subitement fait plonger dans une succession rapide d'expériences religieuses. Dans la perspective dessinée par le cas de Pauline, les ressources religieuses apparaissent comme des ressources permettant de surmonter une cassure, plutôt que de revendiquer une rupture. Dans les deux cas il s'agit, comme le dirait Anthony Giddens, d' " assurer la continuité du récit " (to keep a particular narrative going) (1991: 54) - donc de rendre cohérent un trajet biographique personnel. 


\section{Conclusion}

Les cas que nous venons d'analyser exemplifient quatre manières différentes d'utiliser le bouddhisme : comme centre donnant sens à une vie déjà structurée, comme vision englobant la totalité de la vie, comme complément indispensable à la foi chrétienne, ou comme un moyen parmi d'autres permettant de résoudre le problème central de la mort. Cette exploration n'a nullement permis d'épuiser la diversité des situations imaginables, mais tel n'était pas le but de l'exercice : plus modestement, il s'agissait de renforcer et d'affiner la conception de l'individualisme proposée.

Si l'on revient au cadre théorique de Tönnies et Simmel, il apparaît que la figure de l'individualisme croyant qui a été dessinée est spécifique à la modernité et, probablement - du moins à l'origine - à la modernité occidentale. Nulle trace en effet d'individualisme "dans les liens sociaux » : c'est, fondamentalement, dans la nécessité de donner un fondement symbolique à une trajectoire qui se projette hors des liens que se construit l'individualiste croyant.

Olivier TSCHANNEN

Université de Fribourg

olivier.tschannen@unifr.ch

\section{Bibliographie}

Bajoit Guy et Emmanuel Belin, 1997, Contributions à une sociologie du sujet, Paris, L'Harmattan.

BANCAUD-MAËNEN Florence, 1998, Le roman de formation au XVIII ${ }^{e}$ siècle en Europe, Paris, Nathan.

BELlaH Robert N. et al., 1985, Habits of the Heart: Individualism and Commitment in American Life, New York, Harper \& Row.

Berger Peter L., 1967, The Sacred Canopy: Elements of a Sociological Theory of Religion, Garden City (N.Y.), Doubleday.

Berger Peter L., T. LuCKMAnN, 1966, "Secularization and Pluralism ", International Yearbook for the Sociology of Religion, 2, pp. 73-84.

DumONT Louis, 1966, Homo hierarchicus: le système des castes et ses implications, Paris, Gallimard.

-, 1983, Essais sur l'individualisme: une perspective anthropologique sur l'idéologie moderne, Paris, Seuil.

Durkheim Émile, 1930 [1893], De la division du travail social, Paris, PUF.

Ehrenberg Alain, 1991, Le culte de la performance, Paris, Calmann-Lévy.

ELIAS Norbert, 1993 [1983], Engagement et distanciation : contributions à la sociologie de la connaissance, Paris, Fayard.

GIDDENS Anthony, 1991, Modernity and Self-Identity: Self and Society in the Late Modern Age, Stanford, Stanford University Press. 
I 48 - ARCHIVES DE SCIENCES SOCIALES DES RELIGIONS

Hervieu-Léger Danièle, 1999, Le pèlerin et le converti: la religion en mouvement, Paris, Flammarion.

-, 2001, La religion en miettes, ou la question des sectes, Paris, Calmann-Lévy.

Holstein James A., Jaber F. Gubrium, 2000, The Self we Live by: Narrative Identity in a Postmodern World, New York, Oxford University Press.

Horton Robin, 1993 [1960-1984], Patterns of Thought in Africa and the West: Essays on Magic, Religion and Science, Cambridge, Cambridge University Press.

KIPPELE Flavia, 1998, Was heisst Individualisierung? Die Antworten soziologischer Klassiker, Opladen, Westdeutscher Verlag.

Kuhlman E., 1999, "L'identité comme arme dans le combat pour la vie : le "Nouvel Âge” dans le cadre de la théorie de la civilisation ", Social compass, 46-2, pp. 203-214.

LENOIR Frédéric, 1999, Le bouddhisme en France, Paris, Fayard.

LuCKMANn T., 1990, "Shrinking transcendence, expanding religion? ", Sociological Analysis, 50-2, pp. 127-138.

MARIE Alain, (dir.), 1997, L'Afrique des individus: itinéraires citadins dans l'Afrique contemporaine (Abidjan, Bamako, Dakar, Niamey), Paris, Karthala.

Michon Pierre, 1999, Éléments pour une histoire du sujet, Paris, Éditions Kimé.

MoOre Laurence R., 1994, Selling God: American Religion and the Marketplace of Culture, Oxford, Oxford University Press.

Morris Colin, 1987 [1972], The Discovery of the Individual: 1050-1200, Toronto, University of Toronto Press.

NISBET Robert, 1984 [1966], La tradition sociologique, Paris, PUF.

Pelletier Denis, 2002, La crise catholique : religion, société, politique en France (19651978), Paris, Payot.

SCHNEEBAum Tobias, 1969, Keep the River on your Right, New York, Grove Press.

TSCHANNEN Olivier, 1998, "Religion as a resource for the creation of personal identity in a secularized context ", in Laermans R., Wilson B., Billiet J., Secularization and Social Integration: Papers in Honor of Karel Dobbelaere, Leuven, Leuven University Press, pp. 253-260. 


\section{Résumé}

Dans un paysage religieux devenu pluraliste, comment analyser concrètement les usages individuels auxquels se prête l'offre religieuse? À partir de Tönnies et de Simmel, on peut définir l'individualisme moderne comme le produit d'une rupture avec le milieu socialisateur, rendant possible la projection dans un avenir personnel. Dans ce mouvement, la religion peut offrir des ressources pratiques dans la résolution de "problèmes" apparus dans la trajectoire biographique. Une relecture de cas présentés dans une étude de Frédéric Lenoir sur le bouddhisme en France permet de proposer une exemplification idéal-typique de tels usages, sur le schéma problème I trajectoire / solution, et d'affiner les critères permettant de qualifier différentes formes d'individualisme croyant.

Mots-clés : Individualisme, pluralisme, usage, biographie, appropriation.

\section{Abstract}

In the contemporary pluralistic religious landscape, how should the individual uses to which religion is put be analysed? In line with Tönnies and Simmel, modern individualism can be defined as the product of a break-up from the socializing milieu, which makes the projection in a personal future possible. In this movement, religion can offer practical resources for the resolution of "problems" appearing in the biographical trajectory. On the basis of a second-hand analysis of cases presented in a study on Buddhism in France by Frédéric Lenoir, this paper proposes an idealtypical exemplification of such uses, on the general schema problem / trajectory / solution, and a revision of the analytical criteria for an understanding of different forms of religious individualism.

Key words : Individualism, pluralism, use, biography, appropriation.

\section{Resumen}

En un paisaje religioso que se ha vuelto pluralista, ¿cómo analizar concretamente los usos individuales a los que se presta la oferta religiosa? A partir de Tönnies y de Simmel, se puede definir el individualismo moderno como el producto de una ruptura con el medio socializador, que vuelve posible la proyección de un futuro personal. En este movimiento, la religión puede ofrecer recursos prácticos en la resolución de "problemas" aparecidos en la trayectoria biográfica. Una relectura de los casos presentados en un estudio de Frédéric Lenoir sobre el budismo en Francia nos permite proponer una ejemplificación ideal-típica de tales usos, sobre el esquema problemal trayectorial solución, y de afinar los criterios que permiten calificar diferentes formas de individualismo creyente.

Palabras clave: Idividualismo, pluralismo, uso, biografía, apropriación. 